\title{
Research on Maximum Power Point Tracking Method for Grid- Connected Photovoltaic Power Generation System
}

\author{
Ying Zhang1 \& Junhai Jiang 2 \\ 1 Mechanical and Electrical Engineering ,Jiangsu food and drug of Career Technical College, \\ Huaian ,223001, China. \\ 2Department of omputer Science and Technology,Changchun University, Jilin, 130000, China
}

Keywords Grid-connected photovoltaic power generation, maximum power point, tracking algorithm, controller.

\begin{abstract}
Aiming at the solar photovoltaic characteristics and the lower transformation efficiency problem in grid-connected photovoltaic power generation, a maximum power point tracking (MPPT) algorithm is put forward. According to the changes of amplitude and frequency of power-grid voltage, the proposed algorithm can calculate the correction value of maximum power point, which will be stacked with the maximum power obtained by means of perturbation and observation method, and then the MPPT control is realized. Simulation experiment results present that, compared with the traditional MPPT method using perturbation observation, the proposed method can avoid misjudgment, guarantee the shorter time for maximum power point tracking, and reduce the shock around the maximum power point. Moreover, it can effectively suppress the influences of instable factors on the maximum power point, and meet the optimal power point for stable operation of power grid.
\end{abstract}

\section{Introduction}

With the improvement of science and technology, the level of energy utilization has huge increase. For instance, the use of new energy such as solar, wind or geothermal, not only reduces the usage of the traditional energy such as coal, oil and natural gas, but also reduces the damage to the environmental caused by the use of the traditional energy and protect the environment in which people live. Solar, as a renewable energy source, has found wide application because of the characteristics such as cleanability, reproducibility, economy. The solar use contains photovoltaic, heat and light chemical. It is a kind of energy conversion process. As a kind of solar use, photovoltaic power generation converts solar energy to electrical energy, and is a new power generation mode. This mode features simple structure, clean and safe, ease maintenance.

At present, the development of photovoltaic power generation is taking off in the world, where Germany and Japan possess the fastest growing speed. According to the correlative statistic data, until 2010, the quantity of photovoltaic power generation in Europe has reached to 3.0GW, meanwhile the quantity of photovoltaic power generation in Japan has reached to 4.8GW. In China, the Electrical Power Research Institute predicts that, the quantity of photovoltaic power generation will reach to 30GW until 2020[1-3]. However, it still possesses smaller proportion in the whole power generation of China. The pivotal reason is that, the cost of photovoltaic power generation is much higher, which is greatly related to the lower efficiency in photovoltaic power generation.

This paper synthetically considers the instable factors of power grid in the period of gridconnected power generation, and carries out simulations on the effect of maximum power point tracking (MPPT) in the period of grid-connected power generation. The simulation results show that, the MPPT method proposed in this paper possesses higher tracking accuracy, and can avoid the misjudgment phenomenon by using perturbation and observation method when the power grid is not stable. Moreover, the fluctuation is smaller, and the control effect is better. 


\section{Photovoltaic Cell Equivalent Circuit and Characteristics}

\section{The Equivalent Circuit of Photovoltaic Cell.}

Based on the characteristics of photovoltaic cell, the photovoltaic cell model is equivalent into the conversion semiconductor equipment between solar energy and electric energy. The photovoltaic array is equal to a diode with ultrathin PN section, which is parallel with the light side. It can be regarded as the complex of a current source, a series resistance and a parallel diode [4]. Furthermore, the equivalent circuit is illustrated in Fig. 1.

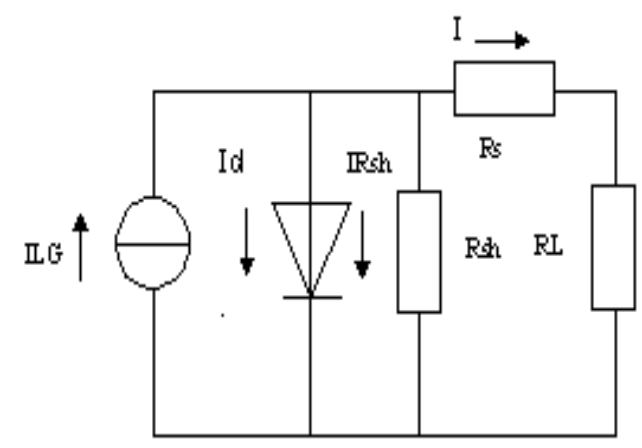

Fig. 1 The equivalent circuit

In Fig. 1, ILG denotes the current of photovoltaic cell; Rsh denotes the equivalent parallel resistance; Rs denotes the equivalent series resistance, which constructs the equivalent current source of photovoltaic array by combining ILG; Id denotes the working current of diode (dark current); IRsh denotes the leakage current; RL denotes the loads; I denotes the output current of photovoltaic array. According the physical definition about photovoltaic, the characteristic equations of the photovoltaic output can be described as

$$
\begin{aligned}
& I=I_{L G}-I_{d}-I_{R s h} \\
& =I_{L G}-I_{\text {os }}\left\{\exp \left[\frac{q}{A K T}(V+I R s)\right]-1\right\}-\frac{V+I R s}{R s h}
\end{aligned}
$$

Where $\mathrm{V}$ denotes the output voltage of the photovoltaic battery, $\mathrm{T}$ represents absolute temperature, $\mathrm{K}$ values $1.38 \times 10^{-23} \mathrm{~J} / \mathrm{K}$, q describes unit charge $\left(1.6 \times 10^{-19} \mathrm{C}\right)$, $\mathrm{A}$ is the ideal factor of diode, $\mathrm{I}_{\mathrm{os}}$ is the saturation current, $\mathrm{I}_{\mathrm{LG}}$ and $\mathrm{I}_{\mathrm{os}}$ are related to $\mathrm{T}$ which denotes the surface temperature of solar cells. The relation between $\mathrm{I}_{\mathrm{LG}}, \mathrm{I}_{\mathrm{os}}$ and $\mathrm{T}$ is shown as

$$
\begin{gathered}
I_{L G}=\left[I_{S C R}+k_{1}(T-298)\right] \frac{\lambda}{1000} \\
I_{o s}=I_{o r}\left[\frac{T}{T r}\right]^{3} \exp \left[\frac{q E_{G O}}{B K}\left(\frac{1}{T r}-\frac{1}{T}\right)\right]
\end{gathered}
$$

Where $\mathrm{I}_{\mathrm{SCR}}$ describes the short-circuit current of solar cells in the standardized temperature and sunshine intensity, k1 describes temperature coefficient, B denotes the ideal factor, EGO is the band gap width of semiconductor material. From above formulas, sunshine and temperature are the major factors to the current of solar cells[5-6]. it is difficulty to solve the formulas, so PV-array model is simplified of considering the accuracy in the actual application processed. The simplified theoretical model of volt ampere properties was derived as

$$
\begin{array}{r}
I=I_{s c}\left\{1-C_{1}\left[\exp \left(\frac{V}{C_{2} V_{o c}}-1\right)\right]\right\} \quad \text { (4) } C_{1}=\left(1-\frac{I_{m}}{I_{s c}}\right) \exp \left(-\frac{V_{m}}{C_{2} V_{o c}}\right) \\
C_{2}=\left(\frac{V_{m}}{V_{o c}}-1\right)\left[\ln \left(1-\frac{I_{m}}{I_{s c}}\right)\right]^{-1}
\end{array}
$$


Considering the effect of environment factor on photovoltaic volt ampere properties, the value of current and voltage in the new environment is calculated on the basis of reference temperature and the value of voltage and current in the sunshine. Through the simplified model, we can get output characteristic of photovoltaic array according to the value of Vm, Im, Voc, Isc at maximum power point.

\section{The Output Characteristics Curve of Photovoltaic Cell.}

Based on the simplified model of photovoltaic array, the simulation module of photovoltaic cell is created in Matlab/Simulink. The corresponding model parameters are shown as: the open-circuit voltage is $22.5 \mathrm{~V}$, the corresponding voltage and current of maximum power point are $17.8 \mathrm{~V}$ and 3.85A respectively, and the short-circuit current is $4.5 \mathrm{~A}$. In the condition of specific temperature $\left(25^{\circ}\right)$ and light intensity $(1 \mathrm{kw} / \mathrm{m} 2)$, the maximum power output of photovoltaic array exists the exclusive point, which is illustrated in Fig. 2.

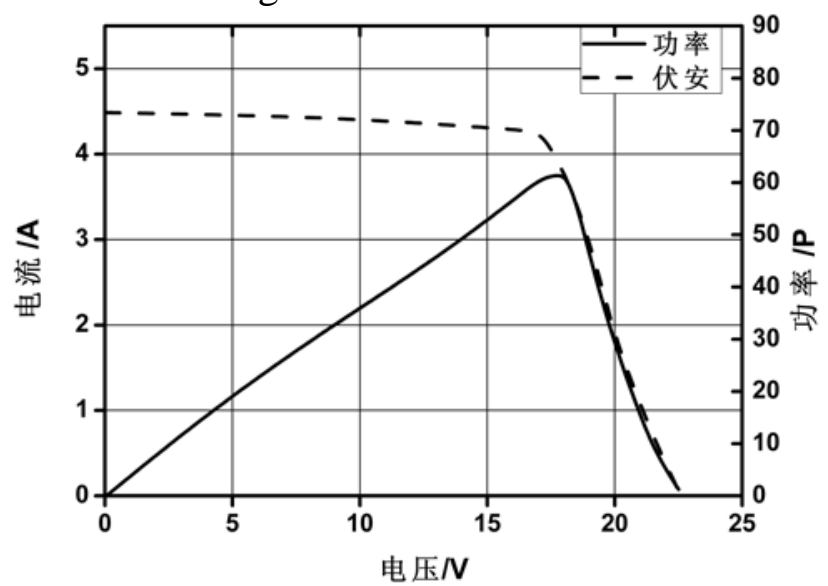

Fig. 2 The curves of Volt-Ampere characteristic and power characteristic of solar cells panel.

From Ref. [7], when the light intensity increases, the output power of solar cell is strengthened. Seen from the Volt-Ampere characteristic curve, when the light intensity weakens, the open-circuit voltage is basically unchanged, meanwhile the short-circuit current decreases. Moreover, the change of open-circuit voltage is influenced by that of temperature. In detail, when the temperature increases, the open-circuit voltage decreases, meanwhile the short-circuit current is little changed. Therefore, when the external conditions are changeable, the outputs of photovoltaic array present the nonlinear properties. In order to exert out the maximum effect of photovoltaic cell, the MPPT control is required. Then, the highest efficiency of photovoltaic cell will be realized.

\section{MPPT Algorithm Realization of Photovoltaic System}

\section{MPPT Principle.}

According to the photovoltaic characteristics of solar cell, the maximum power point in photovoltaic characteristic curve needs to be tracked, in order to achieve the best working state [8]. The MPPT principle is that, under the different light intensities and temperatures, the output voltage of solar cell is changed, and then one can ensure the power point corresponding to some voltage is the maximum through seeking for the terminal voltage of solar cell. With respect to the introduced power converter between the power source and loads, it plays the role in coordinating the output voltage of power source and the input voltage of loads, when the power source voltage is changed. In this paper, a buck-boost DC/DC conversion circuit is introduced in order to realize that the uncontrollable input voltage transforms into the controllable output voltage.

\section{Control Algorithm.}

According to perturbation and observation method [9-10], a reference voltage Ua is introduced. Based on the judgment principle, the reference voltage Ua is regulated by comparing the results, Then, the voltage value, which is most close to the maximum power point, can be obtained. Owing to the direct connection between the photovoltaic power generation system and power grid, and considering the randomness and the uncontrollability in the changes of environment factors 
(including electrical equipment, external temperature, light and so on), which can affect the stability of power-grid voltage, the changes of power-grid voltage (including its frequency and amplitude) will be introduced into the process of MPPT, in order to ensure the stability of power-grid voltage. The detailed realization process of algorithm is shown as follows.

Step 1: Phase-locked calculation is carried out on the voltage UG and the frequency $\mathrm{f}$ of power grid.

Step 2: According to the voltage UG and the frequency $\mathrm{f}$, the corresponding power correction value $\Delta \mathrm{P}$ is obtained. In the process for calculating $\Delta \mathrm{P}$, the output current iG of photovoltaic component is considered, and the amplitudes of power-grid current IG are compared. Moreover, $\Delta \mathrm{P}$ is recognized according to the following equations.

$$
\begin{aligned}
& \Delta \mathrm{P}=K_{U}\left(U_{G}-U_{G N}\right) \\
& \Delta \mathrm{P}=K_{f}\left(f-f_{N}\right)
\end{aligned}
$$

When iG is bigger than the grid-connected current IG, we can employ Eq. (7) to calculate $\Delta P$, where UGN denotes the valid value (i.e., the rated voltage of power grid), and KU denotes the correction coefficient of voltage. In contrary, when iG is smaller than the grid-connected current IG, we can calculate $\Delta \mathrm{P}$ mainly by using the rated frequency $\mathrm{fN}$ of power grid and the correction coefficient kf of frequency, which can be carried out by using Eq. (8).

Step 3: $\Delta \mathrm{P}$ is further corrected, and the corrected value $\Delta \mathrm{P}^{*}$ can be obtained by using function (9).

$$
C\left(\mathrm{P}_{\mathrm{N}}, \mathrm{P}_{\mathrm{dc}}\right)=\left(\frac{\mathrm{P}_{\mathrm{N}}}{\mathrm{P}_{\mathrm{dc}}}\right)^{2}
$$

where, PN represents the rated power of photovoltaic output, and Pdc represents the power of DC current.

Step 4: The power Pmax corresponding to the maximum power point obtained by means of perturbation and observation method is stacked with the corrected value $\Delta \mathrm{P}^{*}$. As a result, the final maximum power point corresponding to the maximum power will be acquired.

\section{Experiment Simulation Results}

Using the power system block (PSB) set in Matlab/Simulink, the simulation is carried out on the improved perturbation and observation algorithm proposed in this paper, in order to verify the MPPT effect of grid-connected photovoltaic power generation system.

According to the characteristics of the choice of step size, i.e., too large step size causes lower tracking accuracy, meanwhile too small step size causes longer tracking time, the step size Cp (0.001-0.1) of perturbation and observation method is chosen as 0.01 , which can bring more ideal simulation results [6]. According to the curves of Volt-Ampere characteristic and power characteristic of solar photovoltaic component shown in Fig. 2, the open-circuit voltage is 22.5V, the short-circuit current is $4.5 \mathrm{~A}$, and the maximum power is $60 \mathrm{~W}$. Based on the improved perturbation control algorithm, the simulation model is constructed. When the disturbances of unstable factors are not added in power grid, the MPPT curve is shown in Fig. 3.

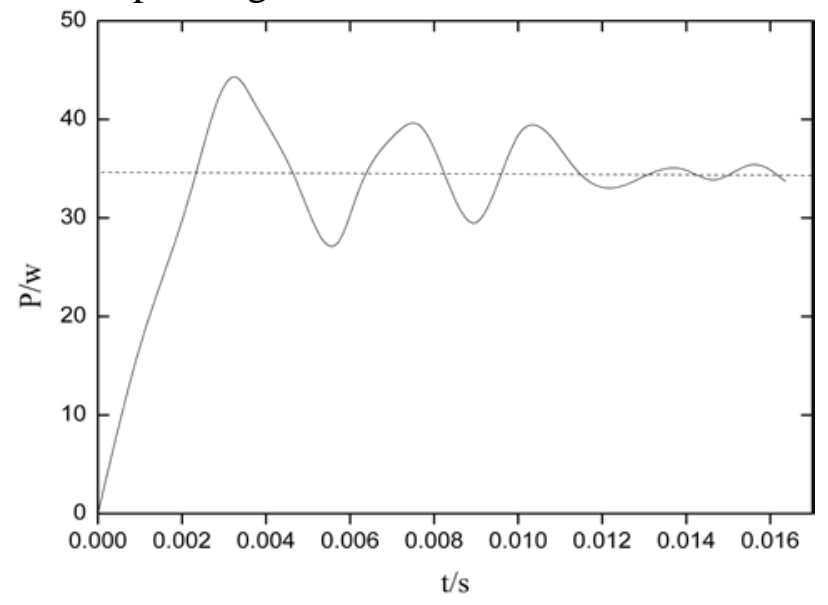


Fig. 3 Results of perturbation and observation method when the power grid is unstable

As seen in Fig. 3, the improved perturbation and observation method possesses better MPPT effect. and the tracking effect is just a come-to-go fluctuation at the maximum power. The reason is that, the perturbation and observation method decides the change of voltage in next step, by comparing the power value corresponding to every step size. Though this process leads to certain energy costs, the total effect is better, and the tracking efficiency can reach to $97.2 \%$. Based on above, the disturbances of unstable factors are added in power grid, and the MPPT curve is obtained and shown in Fig. 4.

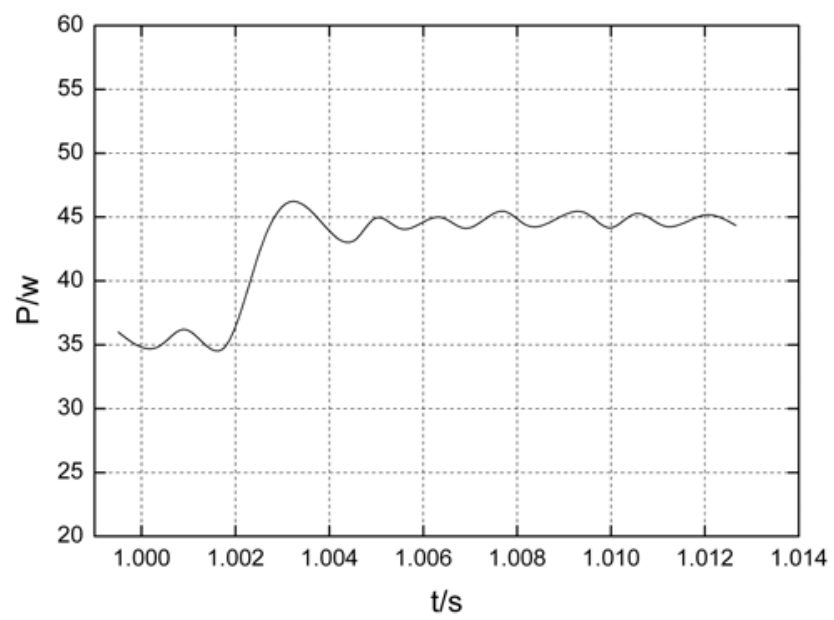

Fig. 4 Results of improved perturbation and observation method when the power grid is unstable

Compared Fig. 4 with Fig. 3, we can see that the system can regulate the output power and exclude the unstable disturbing factors in power grid, and then possesses stable states and smaller fluctuations. When the unstable condition of power-grid voltage occurs, the system can avoid the misjudgment or decrease the probability of misjudgment. From the above figure, we can also see that, when the power-grid voltage is abruptly unstable, the system can fleetly response and then realize the MPPT in a shorter time, which reflects that the dynamic sensitivity of system is better. Synthetically speaking, the improved perturbation and observation method can overcome the influences brought by the unstable factors in power grid, in the process of grid-connected power generation combing the photovoltaic power generation equipments and power grid. Until now, the simulation verification on the effectiveness and the performance advantages of the proposed tracking algorithm has been completed.

\section{Conclusions}

With regard to the grid-connected power generation, the instability of power grid can bring bad impacts on the effect of MPPT. Therefore, this paper puts forward a improved perturbation and observation method with power compensation mode. By means of monitoring the voltage and frequency of power grid and introducing the compensation power with respect to the unstable change of power grid, it can realize the effective tracking for the maximum power point in gridconnected power generation. Simulation results present that, the method combing the power compensation strategy with perturbation and observation idea, avoids the misjudgment phenomenon in the process of MPPT, improves the tracking accuracy, shortens the tracking time, and possesses the better MPPT effect.

\section{References}

[1] Fen Yu, Zhou Lin, Fu Wang, et al. summary of solar array simulator[J].Electrified Transmission, 2011,41(11).1-7.

[2] Hu Bing, Zhang Yanhu, Zhao Wei, et al. Overview of PV power invertertopology and key technology [J]. Power Electronics, 2013,40(3):18-20. 
[3] Liu Dongran, Chen Shuyong, Ma Min, et al. A review on models for photovoltaic generation system[J]. Power System Technology, 2011, 35(8): 47-52.

[4] Z. L. Wang, X. Y. Li, Research on mathematical model of photovoltaic array based on Matlab, Industry and Mine Automation 12 (1012) 40-43.

[5] Kwon M , Kwow B H K, Nam K H. Three-phase photovoltaic system with three-level boosting MPPT control[J]. IEEE Transactions on Power Electronics, 2008, 23(5): 23192327.

[6] Palizban Omid, Mekhilef Saad. Modeling and control of photovoltaic panels base perturbation and observation MPPT method [C] // Proceedings of the 2011 IEEE International Conference on Control System, Computing and Engineering. Penang, Malaysia: IEEE Computer So-ciety, 2011: 393 - 398.

[7] Oscar Lopez Lapena, Teresa Penella Maria, Manel Gasulla. A new MPPT method for lowpower solar energy harvesting[J]. IEEE Transactions on Industrial Electronics, 2010, 57(9): 3129-3138.

[8] G. S. Xie, G. M. Zhang, J. M. Zhang, et al, Application of improved incremental conductance algorithm using variable step in MPPT control of the PV system, Machine Tool and Hydraulics 19 (2011) 38-40.

[9] L. Song, X. D. Wang, W. Liu, et al, Research of perturbation and observation method for MPPT of PV arrays, Semiconductor Optoelectronics 4 (2012) 455-463.

[10] X. J. Guo, W. Q. Kong, J. M. Luo, et al, Solution of photovoltaic cell nonlinear model and research on MPPT, Chinese Journal of Power Source 8 (2014) 1478-1482. 